\title{
PLANTAS HOSPEDEIRAS DE DÍPTEROS MINADORES EM POMAR DE CITROS EM MONTENEGRO, RS
}

\author{
J.P. dos Santos*, F.K. Dal Soglio², L.R. Redaelli²
}

'EPAGRI, Estação Experimental deCaçador, Laboratório deEntomologia, CP 591, CEP 89500-000, Caçador, SC, Brasil. E-mail: janapereira@epagri.rct-sc.br

RESUMO

Este trabalho teve como objetivo verificar a associação do "minador-das-folhas-dos-citros", Phyllocnistis citrella Stainton, 1856 (Lepidoptera: Gracillariidae), com outras plantas hospedeiras, presentes em pomar decitros, a fim deesclarecer aspectos da sua sobrevivência fora dosfluxos de brotação de Citrus spp. efazer um levantamento de insetos minadores que habitam o pomar. $\mathrm{O}$ trabalho foi conduzido em Montenegro, RS, em um pomar orgânico de tangoreiro Murcott. Realizaram-se amostragens quinzenais, de maio de 2003 a mai o de 2004, coletando-se em cada ocasião, todasasfolhas com minas, contidas naáreadelimitadapor umaro de $0,28 \mathrm{~m}^{2}$, queerajogado naslinhasenasentrelinhas de30árvoressorteadas. Duranteo estudo, não foi registradaa presença deP. citrella nas plantas espontâneas do pomar, comprovando-sea sua preferência por espécies de Citrus. Entretanto, registraram-se 15 espécies de dípteros minadores e 15 espécies de plantas hospedeiras, distribuídasemtrêsfamílias.

PALAVRAS-CHAVE:A gromyzidae, Asteraceae, A maranthaceae, Commel inaceae, Convolvulaceae, Lamiaceae.

\section{ABSTRACT}

HOST PLANTS OF DIPTERA LEAFMINERSIN CITRUSORCHARD IN MONTENEGRO, RS, BRAZIL. This study aimed to verify the association of the "citrus leafminer", Phyllocnistis citrella Stainton, 1856(Lepidoptera: Gracillariidae) with other hostplantsthatexistin organiccitrusorchard, in order to explain aspects of its survival out of theC itrus spp flush season , and al so to undertake a survey of leafminers present in theorchard. The work was carried out in M ontenegro, RS, in an organic orchard grown with thehybrid Murcott. Samplings were taken every other week, from May 2003 to May 2004, by collecting on each occasion all themined leavesfound inan areadelimited by $0.28 \mathrm{~m}^{2}$ circlethrown in and between therows of 30 randomly chosen trees. During thestudy, thepresence of P. citrellawasnotreported on spontaneousgrowing plantsin theorchard, and clearly shows its preferencefor C itrus species. However, 15 species of Diptera leafminers and 15 species of host plants bel onging to threebotanical families were recorded.

KEY WORDS: Agromyzidae, Asteraceae, Amaranthaceae, Commelinaceae, Convolvulaceae, Lamiaceae.

\section{INTRODUÇÃO}

Com uma produção de aproximadamente $19 \mathrm{mi}$ Ihões detoneladas métricas nasafra de2003, o Brasil é o maior produtor mundial de frutas cítricas (FAO, 2004). O Rio GrandedoSul éo quinto maior produtor delaranja do país eo quarto maior de limão (BELING et al ., 2004). Em relação às tangerinas éo terceiro maior produtor, responsável por mais de $13 \%$ da produção nacional (FundaçãolBGE, 2002; BeLING et al., 2004). As variedades de tangerineiras Poncã (Citrus reticulata Blanco), Montenegrina (Citrus deliciosa Tenore) e o tangoreiro Murcott (Citrus sinensis(L.) Osbeck xC itrus reticulata Blanco) ocupam amaior partedaárea plantada do Rio Grande do Sul (SouzA, 2001). Estas três variedades estão entreas queapresentam maior tendência à alternância deciclos de produção, podendo apresentar ciclos descontínuos e alterações nas brotações, possibilitando o desenvolvimento de doenças e pragas (SPósito et al., 1998).

2UniversidadeFederal do Rio Grande do Sul, Departamento deFitossanidade, Porto A legre, RS, Brasil. *Bolsistado CN Pq. 
Uma das principais pragas da citricultura em vários países é Phyllocnistis citrella Stainton, 1856 (Lepidoptera, Gracillariidae), conhecidapopularmente como "minador-das-fol has-dos-citros" (H EPPNER, 1993; Cônsol et. al., 1996). Esteinseto atacafol hasnovas das brotações de plantas de citros, ocasionando danos diretos pela redução da área fotossintética (ScHAFFER et al., 1997) e danos indiretos pelo favorecimento da entrada de bactérias, especial mente, a causadora do cancro cítrico, Xanthomonas citri pv. citri (Hasse) Dowson (Heppner, 1993; Chagas et al., 2001).

Phyllocnistis citrella ataca preferencialmenteespé cies deCitrus (Rutaceae)( HeppNer, 1993; Cônsolı, 2001), ou plantas desta mesmafamília, comoF ortunellaspp., M urraya sp., Poncirus sp. eSeverinia sp. (WILLINk et al., 1996; Cônsolı et. al., 1996), além de espécies de Leguminoseae, Loranthaceae, Oleaceae, Lauraceaee plantas ornamentais (Heppner, 1993; Prates et al., 1996; WILLINK et al., 1996). A pesar deter sido relatada a presença de ovos de $P$. citrella em plantas de $V$ itis vinifera L. (Vitaceae), Jasminum sambac (L.) Aiton, Jasminum sp., Dalbergia sissoo Roxb. ex DC. (Leguminoseae), M urraya koenigii (L.) Spreng. (Rutaceae) e Grewia asiatica L. (Tiliaceae), nestas o desenvolvimento larval não foi alcançado (HEPPNER, 1993; Cônsolı, 2001). Já em Salix sp. e M . exotica L. O desenvolvimentolarval foi incompleto (Cônsolı, 2001).

Demodo geral, não há informação sobre o desenvolvimento completo deP . citrella emoutroshospedeiros que não sejam espécies de Citrus. Portanto, o conhecimento de hospedeiros alternativos, além de esclarecer aspectos da sua sobrevivência fora dos fluxos debrotação decitros, permitirátambém o reconhecimento de outras espécies de minadores que podem servir de hospedei ros para parasitóides nativos de P. citrella no Brasil. Este aspecto é de grande valiaparaaimplementação deprogramasdecontrole biológico deP . citrella, tanto pelométododaconservação, quanto pela multiplicação de parasitóides.

Estetrabal ho tevecomo objetivo verificar a associação de P. citrella com outras plantas hospedeiras, presentes em pomar de citros, a fim de esclarecer aspectos da sua sobrevivência fora dos fluxos de brotação de Citrus spp. e fazer um levantamento de insetos minadores que habitam o pomar.

\section{MATERIAL E MÉTODOS}

O trabalho foi desenvolvido no Município de M ontenegro ( $29^{\circ} 68^{\prime} \mathrm{Se} 51^{\circ} 46^{\prime} \mathrm{W}$ ), local izado no Vale do Rio Caí, RS. A topografia da região é levemente ondulada, com menos de $100 \mathrm{~m}$ de altitude, pertencendo à Depressão Central. Os solos são profundose detexturaargilosa (UnidadeBom Retiro). A temperatura média anual é de $19,4^{\circ} \mathrm{C}$, apresentando chuvas abundantes ( $1.537 \mathrm{~mm} / \mathrm{ano}$ ) e bem distribuídas (RodRiguez et al., 1991).

O pomar onde o estudo foi desenvolvido é do tangoreiro M urcott, enxertado sobrePoncirustrifoliata (L.) Raf., comáreade0,6haecercade370 plantas, com 12 anosdeidade. O espaçamentoentreplantaséde3,5m e nas entrelinhas é de $5 \mathrm{~m}$.

Quinzenal mente, demaio de2003a maio de2004, foram realizadasamostragens navegetação quecresce espontaneamente entre as plantas de citros e nas entrelinhas. Para o sorteio dos pontos, as plantas de citros foram numeradas e, através do programa de números aleatórios, BioEstat ${ }^{\circledR}$ (A Yres et al., 2000), em cada ocasião sortearam-se números que corresponderam aos pontosamostrais. Em cadaponto sorteado recolhia-se uma unidade de amostra na linha e outra na entrelinha. Retiraram-se em cada ocasião 60 unidades de amostra que consistiram de todas as fol has com minas presentes num círculo de $0,28 \mathrm{~m}^{2}$, delimitado por um aro de pvc com $60 \mathrm{~cm}$ de diâmetro, adaptação feita do método do quadrilátero, proposto por Soutrwood (1978).

Para retirar a planta inteira utilizou-seuma pá de jardineiro, e quando não era possível retirar toda a planta, retiraram-se os ramos com o auxílio de uma tesoura de poda. As plantas e/ ou ramos foram colocadosindividual mente, emsacos plásticosqueforam acondicionadosem caixadeisopor contendo termogel para o transporte até o laboratório.

No Laboratório de Biologia, Ecologia e Controle Biológico de Insetos, do Departamento de Fitossanidade da Faculdade de A gronomia, da UniversidadeFederal do Rio Grande do Sul (UFRGS), as fol has foram examinadas com o auxílio de microscópio estereoscópio, registrando-seo número delarvas e/ ou pupas deminadores. Asfolhas foramacondicionadas em placas de Petri de $9 \mathrm{~cm}$ de diâmetro e 1,5 $\mathrm{cm}$ de altura e/ ou em caixas gerbox de $11,2 \mathrm{~cm}$ de diâmetro e $3,4 \mathrm{~cm}$ de al tura, que foram mantidas em câmara climatizada (fotofase de $12 \mathrm{~h}, 25^{\circ} \mathrm{C} \pm 1^{\circ} \mathrm{C}$ ) até a emergência dos adultos de minadores e/ ou de parasitóides. Para queas fol has permanecessem túrgidas por um período maior, colocou-se no pecíolo das mesmas, um chumaço de algodão umedecido, queeramolhado diariamenteparafavorecer o desenvolvimento completo dos insetos.

Os dípterosemergidos foram conservados individualmente em tubos "ependorff", contendo álcool $70 \%$, eenviadosà Dra. GracielaValladares (Centrode Investigaciónes Entomológicas deCórdoba, Argentina), para identificação das espécies.

Foram feitas exsicatas das plantas amostradas, procedendo-se a identificação com o auxílio da bibliografia e por comparação com o acervo de plantas do herbário do Departamento de Botânica da UFRGS. 


\section{RESULTADOSE DISCUSSÃO}

$N$ as 27ocasiões deamostragemreal izadas durante o estudo, não se constatou a presença de P. citrella nas plantas espontâneas do pomar. Foram, entretanto, registradas 15 espécies de dípteros minadores da famíliaAgromyzidaee15 espécies deplantas hospedeiras dedípterosminadores, distribuídasem 7famílias (Tabela 1).
O número de espécies de plantas foi crescente ao longo das amostragens, porém, a partir da $20^{a}$ ocasião verificou-se uma estabilização (Fig. 1), indicando que possivel mente, o número apresentado real mentereflita o existentenaárea deestudo. Em relação ao número defamílias deplantas, nãoseobservou mudançaentre

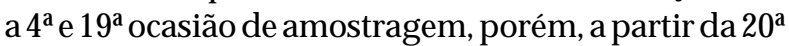
ocasião mais uma família foi registrada e, a partir daí atéofinal dasamostragens, estenúmeronãosealterou.

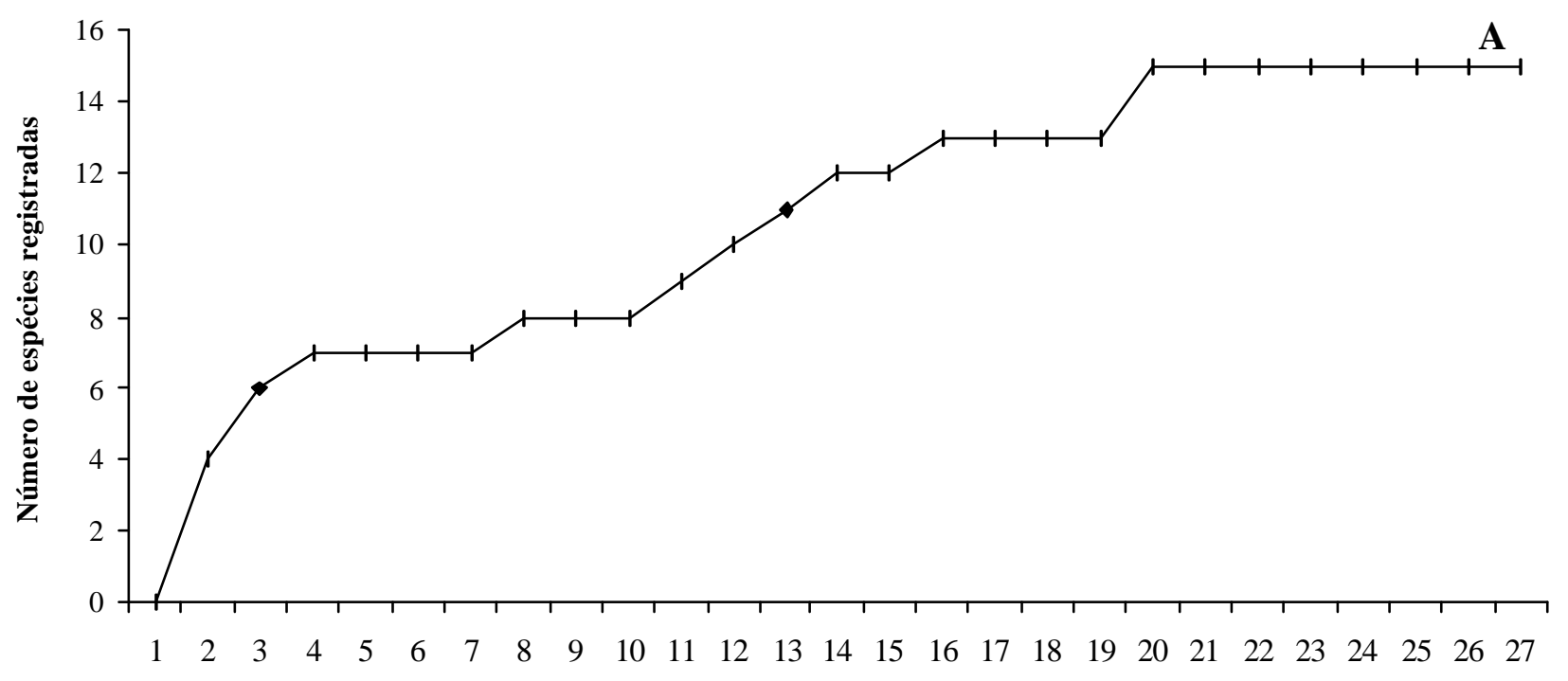

Número de ocasiões de amostragem

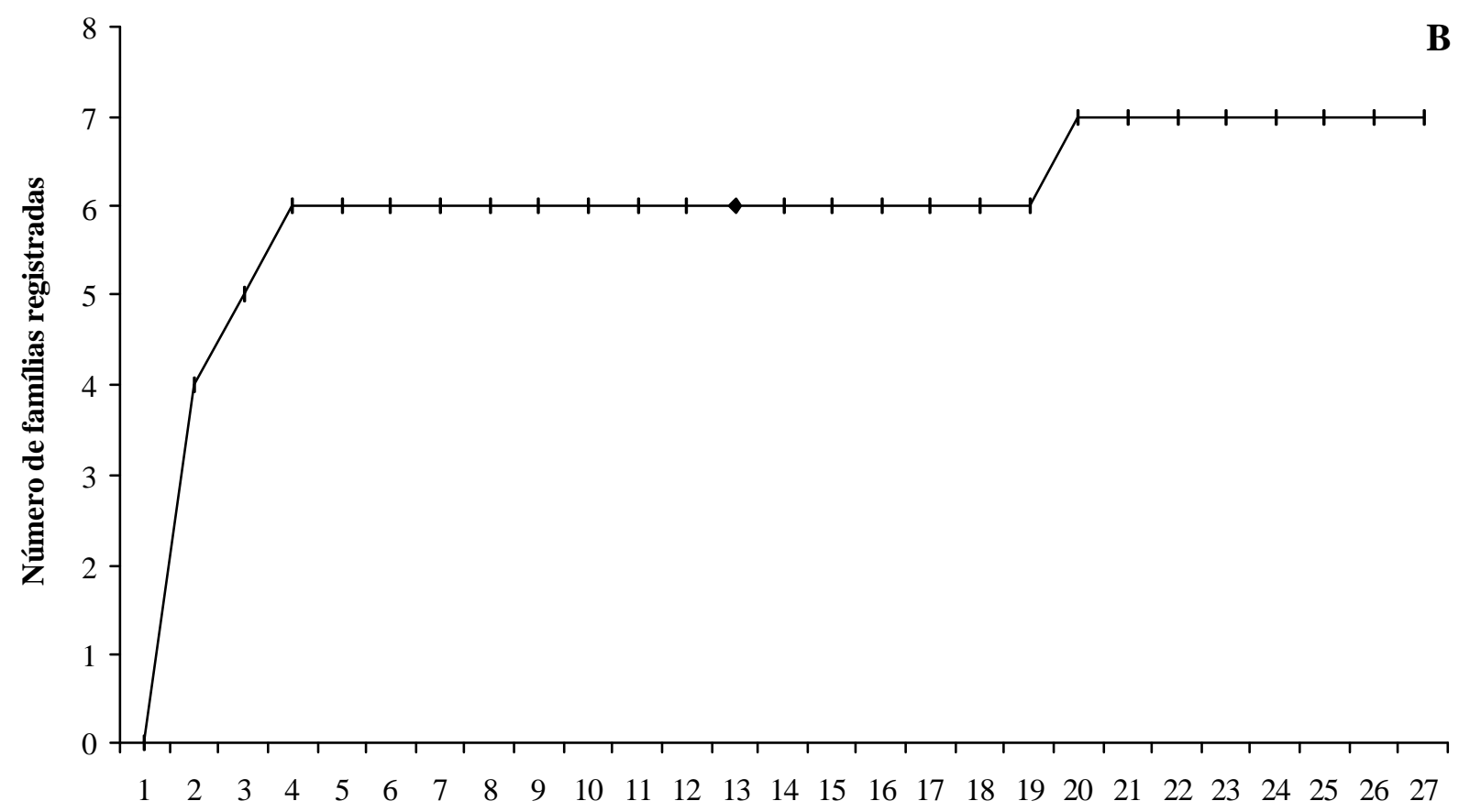

Número de ocasiões de amostragem

Fig. 1- Número cumulativo de (A) espécies e (B) famílias de plantas de crescimento espontâneo obtidas em sucessivas amostragens em pomar do tangoreiro Murcott, em Montenegro, RS (maio de 2003 a maio de 2004). 
Tabela 1- Famílias, espécies enomes comuns de plantas decrescimento espontâneo erespectivas famílias eespécies de dípteros minadores amostrados em pomar do tangoreiro Murcott, em M ontenegro, RS (maio de 2003 a maio de 2004).

\begin{tabular}{|c|c|}
\hline $\begin{array}{l}\text { Plantahospedeira } \\
\text { Família/ Espécie/ Nomecomum }\end{array}$ & DípterominadorFamília/ Espécie \\
\hline $\begin{array}{l}\text { Asteraceae } \\
\text { Baccharis anomala DC., } 1836 \\
\text { (cambará-de-cipó) }\end{array}$ & $\begin{array}{l}\text { Agromyzidae } \\
\text { Calycomyza sp. 1; Liriomyza sp. 1* }\end{array}$ \\
\hline $\begin{array}{l}\text { Baccharis punctulata DC., } 1836 \\
\text { (cambará-cheiroso) }\end{array}$ & Liriomyza sp. 1 \\
\hline $\begin{array}{l}\text { Bidens pilosa L., } 1753 \\
\text { (picão, picão-preto, picão-campo, pico-pico) }\end{array}$ & Calycomyza sp. 2 \\
\hline $\begin{array}{l}\text { Conyza bonariensis (L.) Cronquist, } 1943 \\
\text { (buva, voadeira) }\end{array}$ & Liriomyza sp. 2 \\
\hline $\begin{array}{l}\text { Erechtites valerianifolia (Wolf) DC., } 1837 \\
\text { (capiçoba, capiçoba-vermelha) }\end{array}$ & Calycomyza sp. 3 \\
\hline $\begin{array}{l}\text { Eupatorium inulifolium H.B.K, } 1820 \\
\text { (eupatório, cambará) }\end{array}$ & Calycomyza sp. $4^{* *}$ \\
\hline $\begin{array}{l}\text { M ikania micrantha H.B.K, } 1820 \\
\text { (guaco, micania, bejuco, charrua) }\end{array}$ & $\begin{array}{l}\text { Liriomyza mikaniae Spencer, 1977; } \\
\text { Calycomyza mikaniae Spencer, } 1973\end{array}$ \\
\hline $\begin{array}{l}\text { A maranthaceae } \\
\text { Iresine diffusa Humb. \& Bonpl. ex Willd, } 1806 \\
\text { (bredinho-difuso) }\end{array}$ & Liriomyza sp. 3 \\
\hline $\begin{array}{l}\text { Commelinaceae } \\
\text { Commelina diffusa Burm. f., } 1758 \\
\text { (trapoeraba, rabo-de-cachorro, andaca, maria-mole) }\end{array}$ & Liriomyza commelinae Frost, 1931 \\
\hline $\begin{array}{l}\text { Convolvulaceae } \\
\text { I pomoea cairica (L.) Sweet, } 1827 \\
\text { (corda-de-viola, campainha, corriola, jetirana, enrola-semana) }\end{array}$ & Calycomyza ipomoea (Frost, 1931) \\
\hline $\begin{array}{l}\text { I pomoea purpurea (L.) Roth, } 1787 \\
\text { (campainha, corriola, corda-de-viola, jetirana) }\end{array}$ & Calycomyza ipomoea" \\
\hline $\begin{array}{l}\text { Lamiaceae } \\
\text { Hyptis mutabilis (Rich.) Briq., } 1896 \\
\text { (sambacaitá, bamburral) }\end{array}$ & Calycomyza sp. $5^{\text {*k* }}$ \\
\hline $\begin{array}{l}\text { Sida urens L., } 1759 \\
\text { (guanxuma, vassourinha) }\end{array}$ & Calycomyza malvae (Burgess, 1880) \\
\hline $\begin{array}{l}\text { Sida rhombifolia L., } 1753 \\
\text { (guanxuma, guaxuma, guaxima, guanxuma-preta) }\end{array}$ & Calycomyza sidae Spencer, 1973 \\
\hline $\begin{array}{l}\text { Poaceae } \\
\text { Brachiaria decumbens Stapf, } 1919 \\
\text { (capim braquiária) }\end{array}$ & A gromyza sp." \\
\hline
\end{tabular}

*Provavelmente trata-se deLiriomyza baccharidis Spencer, 1963; **Provavel mentetrata-se de Calycomyza eupatorivora Spencer, 1973, *** Provavel mente trata-se de Caly comyza hyptidis Spencer, 1966; Não emergiram adultos.

EmAsteraceaeforam constatadasB accharisanomala DC., 1836, Baccharis punctulataDC., 1836, Bidens pil osaL., 1753, Conyza bonariensis (L.) Cronquist, 1943, Erechtites valerianifolia (Wolf) DC., 1837, Eupatorium inulifolium
H.B.K, 1820eM ikania micrantha H.B.K, 1820. Estafamília foi a que apresentou o maior número de espécies de plantashospedeiras (sete), com 37,9\% dototal delarvas epupas de dípteros minadores amostradas. 
Registraram-seemB.anomalaasmoscasminadoras Calycomyza sp. 1 e Liriomyza sp. 1. Nesta espécie vegetal, coletaram-se 144 larvas e 11 pupas de Calycomyza sp. 1, verificando-se a emergência de 41 dípteros e 25 indivíduos parasitóides, distribuídos em 3 famílias. Coletaram-se 3 larvas de Li i iomyza sp. $1 \mathrm{em} \mathrm{B}$. anomala e constatou-se a emergência de 2 dípteros e 1 parasitóide. $B$. anomal a foi encontradano local de estudo o ano inteiro. De acordo com BARroso \& BUENO (2002), este arbusto conhecido vulgarmente como "cambará-de-cipó", ocorre no Brasil em Minas Gerais, São Paulo, Paraná, SantaCatarinaeRio Grande do Sul. É também relatado para a Argentina, Paraguai e Uruguai. Floresce de setembro a maio, frutificando rapidamente após a floração.

Em B. punctulata, duas larvas e uma pupa de Liriomyza sp. 1 foram coletadas. Destas, emergiram 1 díptero e 3 indivíduos de parasitóides. Segundo BARROSO \& Bueno (2002), esta planta tem como nomes comuns "vassoura" e "cambará-cheiroso". É um arbusto que floresce de fevereiro a maio, frutificando rapidamente após a floração. No Brasil éencontrada em todos os estados da Região Sul, além de Minas Geraise São Paulo e, naA mérica do Sul, no Paraguai, Argentina eUruguai.

A ssociadas $a B$. pilosa,E. val erianifolia eE. inulifolium verificou-se respectivamente Calycomyza sp. 2, Calycomyza sp. 3 e Calycomyza sp. 4. Em B. pilosa coletaram-se apenas 2 larvas de Calycomyza sp. 2 e não se constatou a emergência de dípteros enem de parasitóides. Já em E. val erianifolia amostraram-se 9 larvas de Calycomyza sp. 3etambém não se registrou a emergência de dípteros, só de 40 indivíduos parasitóides. EmE . inulifol ium 8larvas deC alycomyza sp. 4 foram coletadas e obteve-se 1 díptero.

"Picão", "picão-preto", "picão-campo" e"pico-pico" são alguns dos nomes comuns de B. pilosa, planta herbácea, comaté1,5m dealtura; emboradeocorrência anual, é mais freqüente na primavera e no verão. É nativa da A mérica Tropical, mas mais abundantena AméricadoSul. NoBrasil ocorrecommaisfreqüência comoinvasoradeculturasanuaiseperenes, nasáreas agrícolas das Regiões Sul, Sudeste e Centro-Oeste (KisSmann \& GRoth, 2000a).

E. valerianifolia éuma planta herbácea, de 30 a 120 cm dealtura, conhecidacomo "capiçoba" e"capiçobavermelha"; ocorre anualmente e, na região Sul do Brasil, o ciclo é de 100 a 120 dias, com florescimento de maio a agosto. É nativa da América Tropical e Subtropical; no Brasil ocorre em todo o território e desenvolve-seem regiões com sol o fértil, rico em matéria orgânica (KISSMANn \& Groth, 2000a).

De acordo com Cabrera \& Klein (1989), E. inulifolium éum arbusto queatinge 1 a 2 dealtura, densamente ramoso, conhecido como "eupatório" e "cambará". Floresce desdeoutubro atémaio, com um período menos intenso de novembro a dezembro e outro mais intenso entre março e abril. Ocorre na A mérica Tropical, desde as Antilhas até o centro da Argentina.

Em C. bonariensis registrou-seL iriomyza sp. 2, mas apesar de terem sido coletadas 29 larvas e 1 pupa nesta espécie vegetal, apenas um adulto e 3 parasitóides emergiram. De acordo com Kissmann \& GROTH (2000a), C. bonariensis é uma planta herbácea, anual, nativa da América do Sul, conhecida como "buva" e "voadeira". A germinação ocorrecom maior intensidade no final do outono e no inverno, o ciclo termina na primavera ou no verão. É infestante em culturas anuais de inverno e em áreas de plantio direto.

Associados a M . micrantha observaram-se 2 espé cies de dípteros, Calycomyza mikaniae Spencer, 1973 e Liriomyza mikaniaeSpencer, 1977. N esta espécievegetal coletaram-se 5 larvas de C. mikaniae, registrandose a emergência de 2 dípteros e 2 indivíduos parasitóides. A lém dessa espécie, foram coletadas 21 larvase 3 pupas deL. mikaniae, dasquaisseobtiveram 3 adultos e seis indivíduos parasitóides. Segundo Cabrera \& Klein (1989), M. micrantha é uma erva perene, volúvel, conhecida como "guaco", "micania", "bejuco" e "charrua". Floresce e frutifica esporadicamente, durante todo o ano, preferencial menteno outono (marçoamaio) esedesenvolvebememambientes ensolarados, terrenos recém-abandonados, capoeirões eorla dematas. Ocorredesdeo México até a Argentina, tendo sido introduzida da Ásia (Indonésia e llhas do Pacífico) (CABRERA et al., 1996).

Em Malvaceae, duas espécies de plantas, Sida urens L., 1759 e Sida rhombifolia L., 1753 foram registradas. Associadas a $\mathrm{S}$. urens constatou-se Calycomyza malvae(Burgess, 1880); coletaram-senestaplanta, 118larvase6pupas, obtendo-se17dípteros e 25 indivíduos parasitóides. Já em S. rhombifolia amostraram-seapenas duas larvas enão seobservaram adultos ou parasitóides, porém, pelo formato da minaprovavel menteo minador trata-sedeC alycomyza sidae Spencer, 1973. Deacordo com VALLADARES(informação verbal), C. mal vaefaz minas lineares eamarelas, jáC. sidaefaz minas finasquesetransformam em uma câmara pequena "bloth".

S. urens é uma planta anual ou bienal, geral mente prostrada, atingindo até $80 \mathrm{~cm}$ de altura, conhecida vulgarmente como "guanxuma" e "vassourinha". No Brasil podeser encontrada na maior parte do território, tolera solos arenososeácidos, masseu desenvolvimento é maior em solos bem estruturados, férteise com pH corrigido; éinfestanteem pastagens eculturas, com limitada significação KISSMANn \& GROTH, 2000b).

S. rhombifolia éumaplantaperene, eretacomaté60 cm de altura, porém, em condições ideais, pode se 
tornar semi-arbustivaatingindo até 1,5m dealtura. É comumente chamada de "guanxuma", "guaxuma", "guaxima", "guanxuma-preta", "malva-preta" e "vassourinha". É nativa do continenteamericano eno Brasil ocorre em todas as regiões, infestante em pastagens, áreas abandonadas e diversas culturas (Kissmann \& Groth, 2000b).

Em Convolvulaceae também foram registradas duas espécies de plantas hospedeiras deC alycomyza i pomoea (Frost, 1931), I pomoea cai rica (L.) Sweet, 1827 e I pomoea purpurea (L.) Roth., 1787. Em I. cairica amostraram-se 110 larvas e 3 pupas, de onde se obtiveram 17 dípteros e 30 parasitóides. Já em I. purpurea coletou-seapenas 1 larva e não severificou a emergência de adulto ou parasitóide.

I. cairica éuma herbácea volúvel, perene, conhecida por "corda-de-viola", "campainha", "corriola", "jetirana" e "enrola-semana". É nativa da África Tropical e no Brasil está amplamente distribuída. O florescimento é indeterminado e contínuo. É infestante, especialmente em culturas perenes (Kissmann \& Groth, 2000a).

Segundo descrição de Kissmann \& Groth (2000a), I. purpu rea éuma planta herbácea, conhecida popularmente como "campainha", "corriola", "corda-deviola" e "jetirana". Sua ocorrência é anual e prefere solos trabalhados, férteis e com boa umidade. É originária da América Tropical e Subtropical, porém, está amplamente distribuída pelo mundo. No Brasil se encontra em todos os estados e é muito utilizada como ornamental, mas podeser infestante em culturas anuais.

Em cada uma das seguintes famílias registrou-se apenas 1 espécie de planta hospedeira de díptero minador: Commelinaceae (Commelina diffusaBurm.f., 1758); Lamiaceae (H yptis mutabilis (Rich.) Briq.); A maranthaceae (resine diffusa Humb. \& Bonpl. ex Willd, 1806) e Poaceae (Brachiaria decumbens Stapf).

C. diffusa foi a principal hospedeira das larvas e pupas de Liriomyza commelinae Frost, 1931, com 81 larvas e 43 pupas amostradas, das quais, obtiveramse50dípterose31 parasitóides.C. diffusaéumaplanta herbácea, anual em regiões temperadas e perene em tropicais, conhecida como "trapoeraba", "rabo-decachorro", "andaca" e "maria-mole".

$\mathrm{Em} \mathrm{H}$. mutabilis foram constatadas 13 larvas e 3 pupas de Calycomyza sp. 5, das quais emergiram 3 dípterose2indivíduos parasitóides. KISSMANN\&GROTH (2000b) comentam queH . mutabilis, conhecida como "sambacaitá" e "bamburral", é uma planta subarbustiva, anual, nativado continenteamericano, ocorre em todas as regiões do Brasil, sendo a espécie mais freqüente da região Sul, encontrada na beira de matas e em terrenos abandonados.

Eml . diffusa foram coletadas 7 larvas deL iriomyza sp. 3eemergiram 2 dípteros. Segundo SMITH \& Downs
(1972), I. diffusa é conhecida popularmente como "bredinho-difuso", tem ocorrência anual e floresce o ano inteiro. É nativa da América Tropical e pode ser encontradaemterrenosabandonados, capoeiras, beira de estradas, orla de matas, beira derios, matas semidevastadas e muito abertas.

Foram amostradas em $B$. decumbens três larvas de A gromyza sp.; não se verificou a emergência de moscas, porém, registraram-se dois indivíduos parasitóides. De acordo com Kissmann \& GROTH (2000c), B. decumbens éuma planta perene, nativa do continenteafricano, chamadacomumentede"capimbraquiária", éuma excel enteforrageira, boa para proteger o solo contra os efeitos da erosão.

No Brasil, várias espécies de agromizídeos são relatadascomo pragas deimportânciaagrícola, principalmente as incluídas no gênero Liriomyza. L. huidobrensis Blanchard, 1926 é referida como importante praga da cultura da batata, em São Paulo (CRUZ et al., 1988) eem Minas Gerais (Pereira et al ., 2002). Em São Paulo, Liriomyza spp. é referida como uma das principais pragas em cultivos de cebola, beterraba e batata (DiAs et al., 1997) e L. trifolii (BURGESs, 1880) em tomateiro (Costa et al., 2002).

N esteestudo, registrou-seuma grandediversidade de espécies de dípteros minadores em plantas de crescimento espontâneo. N a bibliografia, estas espé cies não são relatadas como pragas em plantas de importância agrícola, pois são minadores que apresentaramgrandeespecificidadeàs plantas hospedeiras, fato que corrobora com observações de SPENCER (1996). A pesar dos dí pteros registrados não apresentarem importância agrícola, estudos com este grupo são importantes para a contribuição do conhecimento da ecologia de populações de insetos minadores.

\section{CONCLUSÕES}

$N$ ão foi registrada a presença de $P$. citrella nas plantas de crescimento espontâneo presentes no pomar, sugerindo uma preferência deste minador por Citrus spp.

A importância das asteráceas como hospedeiras para os dípteros minadoresficou evidenciada, pois 7 das 15 espécies de plantas amostradas eram desta família.

\section{AGRADECIMENTOS}

Ao CNPq, pela bolsa concedida ao primeiro e terceiro autores; ao Biól. Luís Laux, por ter concedido aáreaderealizaçãodoestudo; aoDr. ThomasMichael Lewinsohn (UNICAMP), pelo encaminhamento dos dípterosparaidentificação; aDra. GracielaValladares 
(Centro de Investigaciónes Entomológicas deCórdoba, Argentina), pela identificação dos dípteros minadores; aos professores M.Sc. Valdely Ferreira Knuppi,Dra.Lílian Auler MentzeDra. Ilsi lobBoldrini (Instituto deBiociências, Departamento deBotânica, UFRGS) eao Dr. N el son I vo M atzenbacher (Faculdade de Biociências, Departamento de Biologia-PUC/ RS), pela confirmação das espécies de plantas.

RefERÊnCIAS

Ayres, M.; Ayres Júnior, M.; Ayres, D.L.; Santos, A.S. dos BioEstat 2.0: aplicações estatísticas nas áreas das ciências biológicas emédicas. Belém: SociedadeCívil Mamirauá; Brasília: CNPq, 2000. 272p.

Barroso, G.M. \& Bueno, O.L. Compostas: subtribo Baccharidinae. In: ReITz, R. (Ed.). Flora ilustrada catarinense. Itajaí: Herbário Barbosa Rodrigues, 2002. p.765-1065.

Beling, R.R.; Santos, C.; Kist, B.B.; Reetz, E.; CorrêA, S.; Schembri, M. A nuário brasileiro da fruticultura 2004. Santa Cruz do Sul: Editora Gazeta, 2004. 136p.

Cabrera, A.L. \& Klein, R.M. Compostas (Eupatoriae). In: REITZ, R. (Ed.). Florailustradacatarinense. Itajaí: Herbário Barbosa Rodrigues, 1989. p.649-750.

Cabrera, A.L.; H olmes, W.C.; M cDaniel, S. CompositaellI: Asteroideae, Eupatoriae.FloraD el Paraguay, v.25, p.208273, 1996.

Chagas, M.C.M.das.; Parra,J.R.P.; N amekata,T.;H artung, J.S.; Yамамото, P.T. Phyllocnistis citrella Stainton (Lepidoptera: Gracillariidae) and its relationship with the citrus canker bacterium Xanthomonas axonopodis pv. citri in Brazil. Ecology, Behavior and Bionomics. Neotropical Entomology, v.30, n.1, p.5559, 2001.

Cônsolı, F.L.; Zucchı, R.A.; LoPES, J.R.S. Phyllocnistis citrella Stainton, 1856 (Lepidoptera: Gracillariidae: Phyllocnistinae): alagarta minadora dos citros. Piracicaba: FEALQ, 1996. 39p.

Cônsolı, F.L. Lagarta-minadora-dos-citros, Phyllocnistis citrella (Lepidoptera: Gracillariidae). In: VILELA, E.; ZucchI, R.A.; CANTOR, F. (Coords.) H istórico e impacto das pragas introduzidas no Brasil. Ribeirão Preto: Holos, 2001. p.23-30.

Costa, V.A.; Raga ,A.;Sato, M.E.;Souza FilhoM.F.;Siloto, R.C. Diversidadede parasitóides deLiriomyzatrifolii Burgess em tomateiro na região central do estado deSão Paulo. In:Congresso Brasileiro deEntomologia, 19., 2002, Manaus, AM. Resumos. Manaus: 2002. p.72.

Cruz, C. de A. da; N akano, O.; Berti Filho, E. Ocorrência de A grostocynips clavatus Díaz, 1976(H ym. Eucoilinae) e O pius sp. (Hym. Braconidae), em pupário deliriomyza huidobrensis Blanchard, 1926(Dip. Agromyzidae). In: Simpósio Nacional de Controle Biológico de Pragas e Vetores 1.; Reunião Nacional Sobre a Utilidade de Microorganismos Entomopatogênicos 1., 1988, Rio de Janeiro, RJ. Resumos. Rio de Janeiro: 1988. p.45.
Dias,A.M.P.;P InTO, R.A.;PAiva,P.E.B.;G Ravena, S. Ocorrência de parasitóides em Liriomyza spp. (Diptera: Agromyzidae) e Plutella xylostella (Lepidoptera: Plutellidae) na região de Piedade, SP. In: Congresso Brasileiro DeEntomologia, 16.; Encontro Nacional Dos Fitossanotaristas, 7., 1997, Salvador, BA. R esumos. Salvador: 1997. p.105.

FAO. Production yearbook. Rome: Fao, 2004.

Heppner, J.B. Citrus leafminer, Phyllocnistis citrella, in Florida (Lepidoptera: Gracillariidae: Phyllocnistinae). Tropical Lepidoptera, v.4, n.1, p.49-64, 1993.

FundAçãolBGE (Brasil).P rodução agrícola municipal:culturas temporárias e permanentes - 2002. Brasília, 2002. 88p.

Kissmann, K.G.; Groth, D. Plantas infestantes enocivas. 2.ed. São Paulo:BA SF Brasileira, IndústriasQuímicas, 2000a. v.2, 978p.

Kissmann, K.G.; Groth, D. Plantas infestantes enocivas. 2.ed. São Paulo:BASF Brasileira, IndústriasQuímicas, 2000b. v.3, 726p.

Kissmann, K.G.; Groth, D. Plantas infestantes enocivas. 2.ed. São Paulo: BASF Brasileira, IndústriasQuímicas, 2000c. v.1, 825p.

Prates, H.S.; Nakano, O.; Gravena, S. A minadora das folhas dos citros "P hyllocnistis citrella" - Stainton, 1856. Campinas: CATI, 1996. p.2-8. (Comunicado Técnico, 129).

Pereira, D.I.da P.; Souza, J.C.de;Santa-Cecília, L.V.C.; Reis, P.R.; SouzA, M. DE A. Parasitismo de larvas da moscaminadora Liriomyza huidobrensis Blanchard (Diptera: Agromyzidae) pelo parasitóide 0 pius sp. (Hymenoptera: Braconidae) naculturadabatatacom faixas de feijoeiro intercaladas. Ciência A grotécnica, v.26, n.5, p.955-963, 2002.

Rodriguez, O.; Viégas, F.; Pompeu Júnior, J.; Amaro, A.A. (Coords.) Citricultura brasileira. Campinas: Fundação Cargill, 1991. 492p.

Schaffer, B.; Peña, J.E.; Colls, A.M.; Hunsberger, A. Citrus leafminer (Lepidoptera: Gracillariidae) in lime: assessment of leaf damageand effectson photosynthesis. Crop Protection , v.16, n.4, p.337-343, 1997.

SMITH, L.B. \& Downs, R.J. A marantáceas. In: Reitz, R. (Ed.). Flora ilustrada catarinense. Itajaí: Herbário Barbosa Rodrigues, 1972. p.1-110.

Southwood, T.R.E. E cological methods: with particular reference to thestudy of insect populations. London: Chapman and Hall, 1978. 524p.

SouzA, A.C. Frutas cítricas: singularidades do mercado. Preços A grícolas, p.8-10, 2001.

SPENCER, K.A.F amilyA gromyzidae.Australasian/ Oceanian Diptera Catalog Web Version, 1996. Disponível em:〈http:/ / www.hbs.bishopmuseum.org/ aocat/ agromyzidae.html>. A cesso em: 2 fev. 2005.

Spósıto, M.B.; CAstro,P.R.;A Gustı, M.Alternânciadeprodução em citros. Laranja, v.19, n.2, p.285-292, 1998.

Willink, E.; Salas, H.; Costilla, M.A.El minador delahoja de los cítricos, Phyllocnistis citrella en el NOA. A vance A groindustrial, v.16, n.65, p.15-20, 1996.

Recebido em 10/ 3/ 06 Aceito em 20/ 6/ 06 